\title{
EXPLICIT GRADIENT ESTIMATES FOR MINIMAL LAGRANGIAN SURFACES OF DIMENSION TWO
}

\author{
MICAH WARREN AND YU YUAN
}

\begin{abstract}
We derive explicit, uniform, a priori interior Hessian and gradient estimates for special Lagrangian equations of all phases in dimension two.
\end{abstract}

\section{INTRODUCTION}

In this note, we derive explicit interior a priori Hessian and gradient estimates for the special Lagrangian equation

$$
\sum_{i=1}^{n} \arctan \lambda_{i}=\Theta
$$

where $\lambda_{i}$ are the eigenvalues of the Hessian $D^{2} u$ and $n=2$. Equation (1.1) stems from the special Lagrangian geometry [HL]. The Lagrangian graph $(x, D u(x)) \subset \mathbb{R}^{n} \times \mathbb{R}^{n}$ is called special when the phase or the argument of the complex number $\left(1+\sqrt{-1} \lambda_{1}\right) \cdots\left(1+\sqrt{-1} \lambda_{n}\right)$ is constant $\Theta$, that is, $u$ satisfies equation (1.1), and it is special if and only if $(x, D u(x))$ is a (volume minimizing) minimal surface in $\mathbb{R}^{n} \times \mathbb{R}^{n}$ [HL, Theorem 2.3, Proposition 2.17]. Gradient estimates for the minimal Lagrangian surfaces are then Hessian estimates for the special Lagrangian equation (1.1). When $n=2$, the potential equation (1.1) also takes the equivalent form

$$
\cos \Theta \triangle u+\sin \Theta\left(\operatorname{det} D^{2} u-1\right)=0 .
$$

We state our result in the following.

Theorem 1.1. Let $u$ be a smooth solution to (1.1) with $n=2$ on $B_{R}(0) \subset$ $\mathbb{R}^{2}$. Then the following both hold

$$
\left|D^{2} u(0)\right| \leq C(2) \exp \left[C(2) \max _{B_{R}(0)}|D u|^{2} / R^{2}\right],
$$

or

$$
\left|D^{2} u(0)\right| \leq C(2) \exp \left[C(2) \frac{1}{|\sin \Theta|^{3 / 2}} \max _{B_{R}(0)}|D u| / R\right] .
$$

Date: August 1, 2007.

Y.Y. is partially supported by an NSF grant. 
In the 1950's, Heinz [H2] derived a Hessian bound for the two dimensional Monge-Ampère type equation including (1.2). In the 1990's Gregori [G] extended Heinz's estimate to a gradient bound in terms of the heights of the two dimensional minimal surfaces with any codimension. A gradient estimate for general dimensional and codimensional minimal graphs with certain constraints on the gradients themselves was obtained in [W].

Although it is not clear whether the exponential dependence in our estimates (1.3) and (1.4) is sharp, still it is sharper than the double exponential dependence on $D u$ by Heinz [H2, Theorem 2], [H1, p.263, p.255] and Gregori [G, Theorem 1], when applied to the special Lagrangian equation of dimension two. On the other hand, like our nonuniform estimate (1.4), Heinz's estimate deteriorates as $\Theta$ goes to 0 .

In order to link the dependence of Hessian estimates in Theorem 1.1 to the potential $u$ itself, we have the following.

Theorem 1.2. Let $u$ be a smooth solution to (1.1) on $B_{3 R}(0) \subset \mathbb{R}^{2}$. Then we have

$$
\max _{B_{R}(0)}|D u| \leq C(2)\left[\underset{B_{3 R}(0)}{\operatorname{osc}} \frac{u}{R}+1\right] .
$$

The strategies of our arguments are as follows. The function associated to the volume element of the special Lagrangian graph, namely, $b=\ln$ $\sqrt{\operatorname{det}\left(I+D^{2} u D^{2} u\right)}$, is subharmonic and satisfies a Jacobi inequality. Using a Poincaré type inequality (instead of the usual mean value inequality by Michael and Simon) together with the Jacobi inequality, the maximum of $b$ on an interior region is bounded by the volume of the ball on the minimal surface. Exploiting the divergence form of the volume element of the minimal Lagrangian graphs, we bound the volume in terms of the height of the special Lagrangian graph, which is the gradient of the solution to equation (1.1). In order to push the resulting Hessian estimate (1.4) independent of the phase $\Theta$, we first employ the Lewy rotation technique to obtain a Hessian estimate for small phase $\Theta$ with a constrained height, then combine it with (1.4) to derive (1.3). Further, we obtain the uniform gradient estimate (1.5) independent of the phase $\Theta$ via the same Lewy rotation, which links the corresponding estimates to the ones for harmonic functions.

More involved arguments are needed to obtain Hessian estimates for the special Lagrangian equation (1.1) with $n=3$ and $\Theta \geq \pi / 2$ in [WY2] and [WY3]. The Bernstein-Pogorelov-Korevaar technique was employed to derive Hessian estimates for (1.1) with certain constraints in [WY1]. The problem of Hessian estimates for (1.1) with general phases $\Theta$ and general dimensions remain open to us.

Notation. $\partial_{i}=\frac{\partial}{\partial x_{i}}, \partial_{i j}=\frac{\partial^{2}}{\partial x_{i} \partial x_{j}}, u_{i}=\partial_{i} u, u_{j i}=\partial_{i j} u$ etc., but $\lambda_{1}, \lambda_{2}$ do not represent the partial derivatives. Further, $h_{i j k}$ will denote (the second 
fundamental form)

$$
h_{i j k}=\frac{1}{\sqrt{1+\lambda_{i}^{2}}} \frac{1}{\sqrt{1+\lambda_{j}^{2}}} \frac{1}{\sqrt{1+\lambda_{k}^{2}}} u_{i j k}
$$

when $D^{2} u$ is diagonalized. The constant $C(2)$ will denote various dimensional constants, which do not depend on the phase $\Theta$.

\section{Preliminary inequalities}

Taking the gradient of both sides of the special Lagrangian equation (1.1), we have

$$
\sum_{i, j}^{n} g^{i j} \partial_{i j}(x, D u(x))=0,
$$

where $\left(g^{i j}\right)$ is the inverse of the induced metric $g=\left(g_{i j}\right)=I+D^{2} u D^{2} u$ on the surface $(x, D u(x)) \subset \mathbb{R}^{n} \times \mathbb{R}^{n}$. Simple geometric manipulation of (2.1) yields the usual form of the minimal surface equation

$$
\triangle_{g}(x, D u(x))=0,
$$

where the Laplace-Beltrami operator of the metric $g$ is given by

$$
\triangle_{g}=\frac{1}{\sqrt{\operatorname{det} g}} \sum_{i, j}^{n} \partial_{i}\left(\sqrt{\operatorname{det} g} g^{i j} \partial_{j}\right) .
$$

Because we are using harmonic coordinates $\triangle_{g} x=0$, we see that $\triangle_{g}$ also equals the linearized operator of the special Lagrangian equation (1.1) at $u$,

$$
\triangle_{g}=\sum_{i, j}^{n} g^{i j} \partial_{i j} .
$$

The gradient and inner product with respect to the metric $g$ are

$$
\begin{aligned}
\nabla_{g} v & =\left(\sum_{k=1}^{n} g^{1 k} v_{k}, \cdots, \sum_{k=1}^{n} g^{n k} v_{k}\right) \\
\left\langle\nabla_{g} v, \nabla_{g} w\right\rangle_{g} & =\sum_{i, j=1}^{n} g^{i j} v_{i} w_{j}, \text { in particular }\left|\nabla_{g} v\right|^{2}=\left\langle\nabla_{g} v, \nabla_{g} v\right\rangle_{g} .
\end{aligned}
$$

We begin with some geometric inequalities.

Lemma 2.1. Let $u$ be a smooth solution to (1.1), with phase $\Theta \geq 0$, and $n=2$. Set $b=\ln V=\ln \sqrt{\operatorname{det}\left(I+D^{2} u D^{2} u\right)}$. Then $b$ satisfies

$$
\triangle_{g} b \geq \sin \Theta\left|\nabla_{g} b\right|^{2}
$$

and for $\Theta \geq \pi / 2$,

$$
\triangle_{g} b \geq\left|\nabla_{g} b\right|^{2}
$$


Proof. Assume that $D^{2} u$ is diagonalized at a point $p$. The calculation

$$
\triangle_{g} \ln \sqrt{\left(1+\lambda_{1}^{2}\right)\left(1+\lambda_{2}^{2}\right)}=\left[4+\left(\lambda_{1}+\lambda_{2}\right)^{2}\right]\left(h_{111}^{2}+h_{112}^{2}\right)
$$

follows from [Y1, Lemma 2.1], where we are using the notation $h_{i j k}=$ $\sqrt{g^{i i}} \sqrt{g^{j j}} \sqrt{g^{k k}} u_{i j k}$. Similarly,

$$
\begin{aligned}
\left|\nabla_{g} b\right|^{2} & =\sum_{i=1}^{2} g^{i i}\left(\partial_{i} \ln \sqrt{\operatorname{det} g}\right)^{2} \\
& =\sum_{i=1}^{2} g^{i i}\left(\frac{1}{2} \sum_{a, b=1}^{2} g^{a b} \partial_{i} g_{a b}\right)^{2}=\sum_{i=1}^{2} g^{i i}\left(\sum_{j=1}^{2} g^{j j} \lambda_{j} u_{j j i}\right)^{2} \\
& =g^{11}\left[g^{11} u_{111}\left(\lambda_{1}-\lambda_{2}\right)\right]^{2}+g^{22}\left[g^{11} u_{112}\left(\lambda_{1}-\lambda_{2}\right)\right]^{2} \\
& =\left(h_{111}^{2}+h_{112}^{2}\right)\left(\lambda_{1}-\lambda_{2}\right)^{2}
\end{aligned}
$$

where we used the minimal surface equation (2.1)

$$
g^{11} u_{111}+g^{22} u_{221}=g^{11} u_{112}+g^{22} u_{222}=0 .
$$

With (1.2) in mind, we compute

$$
\begin{aligned}
4+\left(\lambda_{1}+\lambda_{2}\right)^{2}-\sin \Theta\left(\lambda_{1}-\lambda_{2}\right)^{2} & =4+\sigma_{1}^{2}-\sin \Theta\left(\sigma_{1}^{2}-4 \sigma_{2}\right) \\
& =4+\sigma_{1}^{2}-\sin \Theta\left(\sigma_{1}^{2}-4+4 \cot \Theta \sigma_{1}\right) \\
& =(1-\sin \Theta)\left[\sigma_{1}-2 \frac{\cos \Theta}{(1-\sin \Theta)}\right]^{2} \\
& \geq 0 .
\end{aligned}
$$

Accordingly,

$$
\triangle_{g} b-\sin \Theta\left|\nabla_{g} b\right|^{2}=\left[4+\left(\lambda_{1}+\lambda_{2}\right)^{2}-\sin \Theta\left(\lambda_{1}-\lambda_{2}\right)^{2}\right]\left(h_{111}^{2}+h_{112}^{2}\right) \geq 0 .
$$

The Jacobi inequality (2.2) is proved.

For large phase, $\Theta \geq \pi / 2$, the equation (1.1) dictates that both eigenvalues are positive, and one can see easily from (2.4) and (2.5) that (2.3) holds.

In two dimensions, we take advantage of a certain "super" isoperimetric inequality on the level sets of "subharmonic" functions. The resulting Poincaré type inequality can be used in place of the mean value inequality of Michael and Simon in the proof of Theorem 1.1.

Proposition 2.1. Let $f$ be a smooth, positive function on $B_{2}(0) \subset \mathbb{R}^{2}$. Suppose that $f$ satisfies the weak maximum principle: $f$ attains its maximum on the boundary of any subdomain of $B_{2}$. Then

$$
\|f\|_{L^{\infty}\left(B_{1}\right)} \leq \int_{B_{2}}|D f| d x+\int_{B_{2}} f d x .
$$


Proof. Set $\alpha=\int_{B_{2}} f d x$. We may assume $M \triangleq\|f\|_{L^{\infty}\left(B_{1}\right)}>\alpha$. By Sard's theorem, the level set $\{x \mid f(x)=t\} \cap B_{2}$ is $C^{1}$ for almost all $t$ with $\alpha<$ $t<\|f\|_{L^{\infty}\left(B_{1}\right)}$. For such (almost all) $t$, we show that $\{x \mid f(x)=t\} \cap B_{2}$ has length at least 1 in the following. The set $\{x \mid f(x) \leq t\} \cap B_{1}$ is nonempty and satisfies

$$
\left|\{x \mid f(x) \leq t\} \cap B_{1}\right|>\left|B_{1}\right|-1,
$$

otherwise we have a contradiction:

$$
\alpha>\int_{B_{1}} f d x>t\left|\{x \mid f(x)>t\} \cap B_{1}\right|>\alpha .
$$

If any component of $\{x \mid f(x)=t\} \cap B_{2}$ stretches from the interior $B_{1}$ to the boundary $\partial B_{2}$, then the length $\left|\{x \mid f(x)=t\} \cap B_{2}\right|>1$. Otherwise, each component of $\{x \mid f(x)=t\} \cap B_{2}$ which intersects $B_{1}$ must be a closed curve in $B_{2}$, as we are using the fact that $t$ is not a critical value for $f$. From the maximum principle for $f$, it follows that $f \leq t$ inside any such closed curve. By (2.6) and the usual isoperimetric inequality for each of these (finitely many) $C^{1}$ regions where $f \leq t$, we have

$$
\left|\{x \mid f(x)=t\} \cap B_{2}\right| \geq \sqrt{4 \pi\left|\{x \mid f(x) \leq t\} \cap B_{1}\right|}>1 .
$$

Now we proceed as follows. For any $q \geq 1$,

$$
\begin{aligned}
{\left[\int_{B_{1}}\left|(f-\alpha)^{+}\right|^{q} d x\right]^{1 / q} } & =\left[\int_{0}^{M-\alpha}\left|\{x \mid f(x)-\alpha>t\} \cap B_{1}\right| d t^{q}\right]^{1 / q} \\
& \leq \int_{0}^{M-\alpha}\left|\{x \mid f(x)-\alpha>t\} \cap B_{1}\right|^{1 / q} d t \\
& \leq\left|B_{1}\right|^{1 / q} \int_{0}^{M-\alpha}\left|\{x \mid f(x)-\alpha=t\} \cap B_{2}\right| d t \\
& \leq\left|B_{1}\right|^{1 / q} \int_{B_{2}}|D[f(x)-\alpha]| d x,
\end{aligned}
$$

where the last inequality followed from the coarea formula; the second inequality followed from (2.7); and the first inequality followed from the Hardy-Littlewood-Polya inequality for any nonnegative, nonincreasing integrand $\eta(t)$ (cf. [BDM, p.258]):

$$
\left[\int_{0}^{T} \eta(t)^{q} d t^{q}\right]^{1 / q} \leq \int_{0}^{T} \eta(t) d t .
$$

This H-L-P inequality is proved by noting that $s \eta(s) \leq \int_{0}^{s} \eta(t) d t$ and integrating the inequality

$$
q[s \eta(s)]^{q-1} \eta(s) \leq q\left[\int_{0}^{s} \eta(t) d t\right]^{q-1} \eta(s)=\frac{d}{d s}\left[\int_{0}^{s} \eta(t) d t\right]^{q} .
$$


Letting $q$ go to $\infty$, we have

$$
\left\|(f-\alpha)^{+}\right\|_{L^{\infty}\left(B_{1}\right)} \leq \int_{B_{2}}|D(f-\alpha)| d x .
$$

Thus

$$
\|f\|_{L^{\infty}\left(B_{1}\right)} \leq \int_{B_{2}}|D f| d x+\int_{B_{2}} f d x
$$

\section{Proof of Theorem 1.1}

We combine two estimates to obtain a uniform Hessian estimate for any given height bound. The first estimate, which uses the Jacobi inequality, deteriorates as $\Theta \rightarrow 0$. The second estimate holds for small $\Theta$ with constrained height, and follows easily from a standard technique for harmonic functions, combined with a Lewy rotation of coordinates. For simplicity, we assume that $R=4$ and $u$ is a solution on $B_{4} \subset \mathbb{R}^{2}$. By scaling $u\left(\frac{R}{4} x\right) /\left(\frac{R}{4}\right)^{2}$, we still get the estimate in Theorem 1.1.

Case with $\Theta$-dependence. By the symmetry of the equation (1.1), we assume $\Theta>0$. From inequality (2.2) in Lemma 2.1, $b=\ln V$ is subharmonic with respect to the induced metric on $B_{2}$; hence $b$ satisfies the weak maximum principle. We apply Proposition 2.1

$$
\begin{aligned}
\|b\|_{L^{\infty}\left(B_{1}\right)} & \leq \int_{B_{2}}|D b| d x+\int_{B_{2}} b d x \\
& \leq \int_{B_{2}}\left|\nabla_{g} b\right| d v_{g}+\int_{B_{2}} b d x \\
\leq & \left(\int_{B_{2}}\left|\nabla_{g} b\right|^{2} V d x\right)^{1 / 2}\left(\int_{B_{2}} V d x\right)^{1 / 2}+\int_{B_{2}} V d x .
\end{aligned}
$$

Multiplying both sides of the Jacobi equation (2.2) by a non-negative cut-off function $\psi \in C_{0}^{\infty}\left(B_{3}\right)$ with $\psi=1$ on $B_{2}$ and $|D \psi| \leq 1.1$, then integrating, we obtain

$$
\begin{aligned}
\int_{B_{3}} \psi^{2}\left|\nabla_{g} b\right|^{2} d v_{g} & \leq \frac{1}{\sin \Theta} \int_{B_{3}} \psi^{2} \triangle_{g} b d v \\
& =-\frac{1}{\sin \Theta} \int_{B_{3}}\left\langle 2 \psi \nabla_{g} \varphi, \nabla_{g} b\right\rangle_{g} d v_{g} \\
& \leq \frac{1}{2} \int_{B_{3}} \psi^{2}\left|\nabla_{g} b\right|^{2} d v_{g}+2\left(\frac{1}{\sin \Theta}\right)^{2} \int_{B_{3}}\left|\nabla_{g} \psi\right|^{2} d v_{g}
\end{aligned}
$$

It follows that

$$
\int_{B_{2}}\left|\nabla_{g} b\right|^{2} V d x \leq \int_{B_{3}} \psi^{2}\left|\nabla_{g} b\right|^{2} d v_{g} \leq 4 \csc ^{2} \Theta \int_{B_{3}}\left|\nabla_{g} \psi\right|^{2} d v_{g}
$$


Observe that by equation (1.1) or (1.2) the volume element takes a simple form

$$
\begin{aligned}
V & =\sqrt{\left(1+\lambda_{1}^{2}\right)\left(1+\lambda_{2}^{2}\right)}=\left|\left(1+i \lambda_{1}\right)\left(1+i \lambda_{2}\right)\right|=\left|1-\sigma_{2}+i \sigma_{1}\right| \\
& =\frac{\sigma_{1}}{\sin \Theta}=\csc \Theta \triangle u .
\end{aligned}
$$

Hence,

$$
\int_{B_{2}} V d x \leq \frac{C(2)}{\sin \Theta}\|D u\|_{L^{\infty}\left(B_{2}\right)}
$$

and

$$
\begin{aligned}
\left|\nabla_{g} \psi\right|^{2} V & \leq\left(\frac{|D \psi|^{2}}{1+\lambda_{1}^{2}}+\frac{|D \psi|^{2}}{1+\lambda_{2}^{2}}\right) V=|D \psi|^{2}\left(\frac{2+\lambda_{2}^{2}+\lambda_{1}^{2}}{V}\right) \\
& =|D \psi|^{2}\left[2\left(1-\sigma_{2}\right)+\sigma_{1}^{2}\right] \frac{\sin \Theta}{\sigma_{1}}=|D \psi|^{2}\left(2 \cos \Theta+\sigma_{1} \sin \Theta\right)
\end{aligned}
$$

where we used the equation (1.2). We then have from (3.2)

$$
\begin{aligned}
\int_{B_{2}}\left|\nabla_{g} b\right|^{2} V d x & \leq C(2) \csc ^{2} \Theta \int_{B_{3}}\left(2 \cos \Theta+\sigma_{1} \sin \Theta\right) d x \\
& \leq C(2)\left(\csc ^{2} \Theta+\csc \Theta\|D u\|_{L^{\infty}\left(B_{3}\right)}\right) .
\end{aligned}
$$

Thus from (3.1),

$$
\|b\|_{L^{\infty}\left(B_{1}\right)} \leq C(2) \frac{\left(1+\sin \Theta\|D u\|_{L^{\infty}\left(B_{3}\right)}\right)^{\frac{1}{2}}}{\sin \Theta}\left[\frac{\|D u\|_{L^{\infty}\left(B_{2}\right)}}{\sin \Theta}\right]^{\frac{1}{2}}+C(2) \frac{\|D u\|_{L^{\infty}\left(B_{2}\right)}}{\sin \Theta}
$$

that is

$$
\|b\|_{L^{\infty}\left(B_{1}\right)} \leq C(2) \frac{\|D u\|_{L^{\infty}\left(B_{3}\right)}}{\sin \Theta}\left(1+\frac{1}{\sin ^{1 / 2} \Theta\|D u\|_{L^{\infty}\left(B_{3}\right)}^{1 / 2}}\right) .
$$

The estimate (1.4) follows by exponentiating (3.3).

Next, for very large phase, $\Theta>3 \pi / 4$, we adapt the proof of (3.3) to obtain a bound that does not deteriorate as $\Theta \rightarrow \pi$. First we note that from the Jacobi inequality (2.3) the $\Theta$-dependence in $(3.2)$ is no longer needed, and we have

$$
\int_{B_{2}}\left|\nabla_{g} b\right| d v_{g} \leq 4\left(\int_{B_{3}}\left|\nabla_{g} \psi\right|^{2} d v_{g}\right)^{1 / 2}\left(\int_{B_{2}(0)} V d x\right)^{1 / 2} \leq C(2) \int_{B_{3}(0)} V d x .
$$

Using another expression for the volume form

$$
V=\left|1-\sigma_{2}+i \sigma_{1}\right|=|\sec \Theta|\left(\sigma_{2}-1\right)
$$


for $\Theta>\pi / 2$,

$$
\begin{aligned}
\int_{B_{r}(0)} V d x & =\int_{B_{r}(0)}|\sec \Theta|\left(\sigma_{2}-1\right) d x \leq|\sec \Theta| \int_{B_{r}(0)} \sigma_{2} d x \\
& =|\sec \Theta| \int_{B_{r}(0)} \operatorname{div}\left(u_{1} u_{22},-u_{1} u_{21}\right) d x \\
& \leq|\sec \Theta|\|D u\|_{L^{\infty}\left(B_{r}\right)} \int_{\partial B_{r}(0)}\left|D^{2} u\right| d s
\end{aligned}
$$

By the convexity of $u$ for large phase $\Theta>\pi / 2$, we know $\triangle u \geq\left|D^{2} u\right|$, so

$$
\int_{B_{r}(0)} V d x \leq|\sec \Theta|\|D u\|_{L^{\infty}\left(B_{r}\right)} \int_{\partial B_{r}(0)} \triangle u d s .
$$

Integrating the right hand side from $r=3$ to $r=4$, we deduce

$$
\begin{aligned}
\int_{B_{3}(0)} V d x & \leq|\sec \Theta|\|D u\|_{L^{\infty}\left(B_{4}\right)} \min _{r \in[3,4]} \int_{\partial B_{r}(0)} \triangle u d s \\
& \leq|\sec \Theta|\|D u\|_{L^{\infty}\left(B_{4}\right)} \int_{B_{4}(0)} \triangle u d x \leq|\sec \Theta|\|D u\|_{L^{\infty}\left(B_{4}\right)}^{2} .
\end{aligned}
$$

In light of (3.1) and (3.4), we then have for $\Theta>\pi / 2$,

$$
\|b\|_{L^{\infty}\left(B_{1}\right)} \leq C(2)|\sec \Theta|\|D u\|_{L^{\infty}\left(B_{4}\right)}^{2}
$$

and finally

$$
\left\|D^{2} u\right\|_{L^{\infty}\left(B_{1}\right)} \leq \exp \left[C(2)|\sec \Theta|\|D u\|_{L^{\infty}\left(B_{4}\right)}^{2}\right] .
$$

This finishes the estimates with $\Theta$-dependence in Theorem 1.1.

Case without $\Theta$-dependence. In order to prove the Hessian bound (1.3) that does not deteriorate for small $\Theta$, we need the following.

Proposition 3.1. Let $u$ be a smooth solution to (1.1) with $n=2$ and $\Theta \in[0, \pi / 4]$ on $B_{1}(0) \subset \mathbb{R}^{2}$. Suppose that

$$
\|D u\|_{L^{\infty}\left(B_{1}\right)} \leq \frac{1}{8 \sin \Theta}
$$

Then we have

$$
\left|D^{2} u(0)\right| \leq C(2)\left(\|D u\|_{L^{\infty}\left(B_{1}\right)}+1\right) .
$$

Proof. We first find a harmonic representation of $\mathfrak{M}=(x, D u)$ via Lewy rotation (cf. [Y1], [Y2, p.1356]). We take a $U(2)$ rotation of $\mathbb{C}^{2} \cong \mathbb{R}^{2} \times \mathbb{R}^{2}$ : $\bar{z}=e^{-\sqrt{-1} \Theta / 2} z$ with $z=x+\sqrt{-1} y$ and $\bar{z}=\bar{x}+\sqrt{-1} \bar{y}$. Because a $U(2)$ rotation preserves the length and complex structure, $\mathfrak{M}$ is still a special Lagrangian submanifold with the parametrization

$$
\left\{\begin{array}{c}
\bar{x}=x \cos \frac{\Theta}{2}+D u(x) \sin \frac{\Theta}{2} \\
\bar{y}=-x \sin \frac{\Theta}{2}+D u(x) \cos \frac{\Theta}{2}
\end{array} .\right.
$$


In order to show that this parametrization is that of a gradient graph over $\bar{x}$, we show that $\bar{x}(x)$ is a diffeomorphism onto its image. This is accomplished by showing that

$$
\left|\bar{x}\left(x_{a}\right)-\bar{x}\left(x_{b}\right)\right| \geq \frac{1}{2 \cos \Theta / 2}\left|x_{a}-x_{b}\right|
$$

for any $x_{a}, x_{b}$. We assume by translation that $x_{b}=0$ and $D u\left(x_{b}\right)=0$. Now $\theta_{i}>\Theta-\frac{\pi}{2}$, so $u+\frac{1}{2} \cot \Theta x^{2}$ is convex, and we have

$$
\begin{gathered}
\left|\bar{x}\left(x_{a}\right)-\bar{x}(0)\right|^{2}=\left|\bar{x}\left(x_{a}\right)\right|^{2}=\left|x_{a} \cos \frac{\Theta}{2}+D u\left(x_{a}\right) \sin \frac{\Theta}{2}\right|^{2} \\
=\left|x_{a}\left(\cos \frac{\Theta}{2}-\cot \Theta \sin \frac{\Theta}{2}\right)+\left[D u\left(x_{a}\right)+x_{a} \cot \Theta\right] \sin \frac{\Theta}{2}\right|^{2} \\
\geq\left|x_{a}\right|^{2}\left(\frac{\sin \frac{\Theta}{2}}{\sin \Theta}\right)^{2}+\left|D u\left(x_{a}\right)+x_{a} \cot \Theta\right|^{2} \sin ^{2} \frac{\Theta}{2}+2 \frac{\sin ^{2} \frac{\Theta}{2}}{\sin \Theta}\left\langle x_{a}, D u\left(x_{a}\right)+x_{a} \cot \Theta\right\rangle \\
\geq\left|x_{a}\right|^{2}\left(\frac{1}{2 \cos \Theta / 2}\right)^{2} .
\end{gathered}
$$

We see that $\mathfrak{M}$ is a (special Lagrangian) graph over $\bar{x}$ space: $\mathfrak{M}=(\bar{x}, D \bar{u}(\bar{x}))$, where $\bar{u}$ is a smooth function. Let $\bar{\lambda}_{i}$ be the eigenvalues of the Hessian $D^{2} \bar{u}$. Then

$$
\arctan \bar{\lambda}_{i}=\arctan \lambda_{i}-\frac{\Theta}{2} \in\left(-\frac{\pi}{2}+\frac{\Theta}{2}, \frac{\pi}{2}-\frac{\Theta}{2}\right) .
$$

It follows that

$$
\begin{aligned}
\arctan \bar{\lambda}_{1}+\arctan \bar{\lambda}_{2} & =0 \quad \text { or } \\
\triangle \bar{u} & =0 .
\end{aligned}
$$

Moreover, the domain of $\bar{u}, \bar{x}\left(B_{1}\right)$ contains a ball in $\bar{x}$ space with radius $\bar{R}$ at least

$$
\bar{R} \geq \frac{1}{2 \cos \Theta / 2}
$$

around $\bar{x}(0)$.

For the harmonic function $\bar{u}_{\bar{e} \bar{e}}$ with $\bar{e}$ being an arbitrary unit vector in $\bar{x}$ space, the mean value formula implies

$$
\bar{u}_{\bar{e} \bar{e}}(\overline{0})=\frac{1}{\pi \bar{R}^{2}} \int_{\bar{B}_{\bar{R}}} \bar{u}_{\bar{e} \bar{e}} d \bar{x} \leq \frac{2 \pi \bar{R}}{\pi \bar{R}^{2}}\|D \bar{u}\|_{L^{\infty}\left(\bar{B}_{\bar{R}}\right)} \leq 4 \cos \frac{\Theta}{2}\|D \bar{u}\|_{L^{\infty}\left(\bar{B}_{\bar{R}}\right)} .
$$

From the above harmonic parametrization (3.6) of $\mathfrak{M}$, we know

$$
\|D \bar{u}\|_{L^{\infty}\left(\bar{B}_{\bar{R}}\right)} \leq \sin \frac{\Theta}{2}+\cos \frac{\Theta}{2}\|D u\|_{L^{\infty}\left(B_{1}\right)} .
$$

Thus we get

$$
\bar{\lambda}_{i}(\overline{0}) \leq 4 \cos \frac{\Theta}{2}\left[\sin \frac{\Theta}{2}+\cos \frac{\Theta}{2}\|D u\|_{L^{\infty}\left(B_{1}\right)}\right]
$$


From (3.8), we see that

$$
\lambda_{i}(0)=\frac{\bar{\lambda}_{i}(\overline{0})+\tan \frac{\Theta}{2}}{1-\bar{\lambda}_{i}(\overline{0}) \tan \frac{\Theta}{2}} .
$$

Note that $\lambda_{\max }(0) \geq\left|\lambda_{i}(0)\right|$ for $\Theta \geq 0$. It follows that

$$
\begin{aligned}
\left|D^{2} u(0)\right| & \leq \lambda_{\max }(0) \\
& \leq \frac{\tan \frac{\Theta}{2}+4 \cos \frac{\Theta}{2}\left[\sin \frac{\Theta}{2}+\cos \frac{\Theta}{2}\|D u\|_{L^{\infty}\left(B_{1}\right)}\right]}{2 \cos \Theta-1-2 \sin \Theta\|D u\|_{L^{\infty}\left(B_{1}\right)}} \\
& \leq C(2)\left[\|D u\|_{L^{\infty}\left(B_{1}\right)}+1\right]
\end{aligned}
$$

provided that, say, $\left[2 \cos \Theta-1-2 \sin \Theta\|D u\|_{L^{\infty}\left(B_{1}\right)}\right]>0.15$, which is available under our assumption.

We finish the proof of Theorem 1.1 without $\Theta$-dependence. By symmetry, we only consider the cases $0 \leq \Theta<\pi$.

We first consider the small phase $0 \leq \Theta \leq \pi / 4$.

If $\|D u\|_{L^{\infty}\left(B_{3}\right)} \geq \frac{1}{8 \sin \Theta}$, then from the first estimate (3.3) we have

$$
\begin{aligned}
\left\|D^{2} u\right\|_{L^{\infty}\left(B_{1}\right)} & \leq C(2) \exp \left\{C(2) \frac{\|D u\|_{L^{\infty}\left(B_{3}\right)}}{\sin \Theta}\left[1+\frac{1}{\sin ^{1 / 2} \Theta\|D u\|_{L^{\infty}\left(B_{3}\right)}^{1 / 2}}\right]\right\} \\
& \leq C(2) \exp \left[C(2)\|D u\|_{L^{\infty}\left(B_{4}\right)}^{2}\right] .
\end{aligned}
$$

If $\|D u\|_{L^{\infty}\left(B_{2}\right)}<\frac{1}{8 \sin \Theta}$, then Proposition 3.1 (applied at any point in $B_{1}$ ) implies

$$
\begin{aligned}
\left\|D^{2} u\right\|_{L^{\infty}\left(B_{1}\right)} & \leq C(2)\left[\|D u\|_{L^{\infty}\left(B_{2}\right)}+1\right] \\
& \leq C(2) \exp \left[C(2)\|D u\|_{L^{\infty}\left(B_{4}\right)}^{2}\right] .
\end{aligned}
$$

For phases $\pi / 4 \leq \Theta \leq 3 \pi / 4, \quad \sin \Theta$ is bounded away from 0 and (3.3) gives

$$
\begin{aligned}
\left\|D^{2} u\right\|_{L^{\infty}\left(B_{1}\right)} & \leq C(2) \exp \left(C(2)\|D u\|_{L^{\infty}\left(B_{3}\right)}\right) \\
& \leq C(2) \exp \left(C(2)\|D u\|_{L^{\infty}\left(B_{4}\right)}^{2}\right) .
\end{aligned}
$$

For large phase $\Theta \geq 3 \pi / 4$, sec $\Theta$ is bounded and we have from (3.5)

$$
\left\|D^{2} u\right\|_{L^{\infty}\left(B_{1}\right)} \leq C(2) \exp \left(C(2)\|D u\|_{L^{\infty}\left(B_{4}\right)}^{2}\right) .
$$

The proof of estimate (1.3) without $\Theta$-dependence in Theorem 1.1 is complete after a scaling. 


\section{Proof of Theorem 1.2}

By symmetry we assume $\Theta \geq 0$. By scaling $u\left(\frac{R}{3} x\right) /\left(\frac{R}{3}\right)^{2}$, we may assume that $u$ is a solution on $B_{3}(0)$. If $\Theta=0$, then $u$ is harmonic and the linear gradient estimate is standard. Otherwise, using

$$
\arctan \lambda_{i}>\Theta-\frac{\pi}{2}
$$

we can control the gradient of the convex function $u(x)+\frac{1}{2} \max \{\cot \Theta, 0\} x^{2}$ by its oscillation. Thus

$$
|D u(0)| \leq \underset{B_{1}}{\operatorname{osc}} u+\frac{1}{2} \max \{\cot \Theta, 0\} .
$$

The following uses the same rotation argument as in Theorem 1.1 to deal with very small $\Theta$.

Proposition 4.1. Let $u$ satisfy (1.1) with $\Theta \in(0, \pi / 4)$ on $B_{2}(0)$. Suppose that

$$
\underset{B_{2}}{\operatorname{Oac}} u \leq \frac{1}{2 \sin \Theta}
$$

Then

$$
|D u(0)| \leq C(2)\left(\underset{B_{2}}{\operatorname{osc} u+1}\right) .
$$

Proof. We perform a Lewy rotation as before, to obtain a harmonic representation $\mathfrak{M}=(\bar{x}, D \bar{u}(\bar{x}))$ for the original special Lagrangian graph $\mathfrak{M}=$ $(x, D u(x))$ with $x \in B_{2}$. Recentering the new coordinates, we take

$$
\left\{\begin{array}{l}
\bar{x}=x \cos \frac{\Theta}{2}+D u(x) \sin \frac{\Theta}{2}-D u(0) \sin \frac{\Theta}{2} \\
D \bar{u}(\bar{x})=-x \sin \frac{\Theta}{2}+D u(x) \cos \frac{\Theta}{2}
\end{array}\right.
$$

By (3.7) we see that the harmonic function $\bar{u}$ is defined on a ball of radius

$$
\bar{R}=\frac{2}{2 \cos \left(\frac{\Theta}{2}\right)}>1
$$

in $\bar{x}$-space around $\overline{0}$.

From (4.3) and the classical estimate on the derivative of the harmonic function $\bar{u}$, we have

$$
|D u(0)|=\frac{|D \bar{u}(\overline{0})|}{\cos (\Theta / 2)} \leq C(2) \max _{\bar{B}_{1}(\overline{0})}|\bar{u}-\bar{u}(\overline{0})| .
$$

We may assume that $\bar{u}(\overline{0})=0$. The maximum of $|\bar{u}|$ on $\bar{B}_{1}(\overline{0})$ must occur on the boundary, without loss of generality we assume this happens along the positive $\bar{x}_{1}$-axis. Thus we have

$$
\max _{\bar{B}_{1}(\overline{0})}|\bar{u}|=\left|\int_{\bar{x}_{1}=0}^{\bar{x}_{1}=1} \bar{u}_{\bar{x}_{1}} d \bar{x}_{1}\right| .
$$


In the following, we convert the integral of $\bar{u}_{\bar{x}_{1}}$ to one in terms of $u_{x_{1}}$, then recover the oscillation of $\bar{u}$ from that of $u$.

We work on the $x_{1}-y_{1}$ plane in the remaining proof. Under our above assumption, the $\bar{x}_{1}$-axis is given by the line

$$
y_{1}=\tan \left(\frac{\Theta}{2}\right) x_{1}
$$

and the curve $\gamma:\left(x_{1}, u_{1}\left(x_{1}\right)\right)$ with $\left|x_{1}\right|<2$ forms a graph over $\bar{x}_{1}$-axis. Let $l_{0}$ be the line perpendicular to $\bar{x}_{1}$-axis and intersecting the curve $\gamma$ at $\left(0, u_{1}(0)\right)$ along the $y_{1}$-axis. The intersection of $l_{0}$ and the $\bar{x}_{1}$-axis (which is also the origin of the recentered $\bar{x}_{1}-\bar{y}_{1}$ plane) has distance to the origin of $x_{1}-y_{1}$ plane given by

$$
\left|u_{1}(0)\right| \sin \left(\frac{\Theta}{2}\right) \leq\left(\underset{B_{1}}{\operatorname{osc}} u+\frac{1}{2} \cot \Theta\right) \sin \left(\frac{\Theta}{2}\right) \leq 1
$$

by the rough bound (4.1) and the condition (4.2). Now let $l_{1}$ be the line parallel to $l_{0}$ passing through the point $\bar{x}_{1}=1$ along the $\bar{x}_{1}$-axis.

The integral

$$
\int_{\bar{x}_{1}=0}^{\bar{x}_{1}=1} \bar{u}_{\bar{x}_{1}} d \bar{x}_{1}
$$

is the signed area between the $\bar{x}_{1}$-axis and the curve $\gamma$, and lying between the lines $l_{0}$ and $l_{1}$. We convert this to an integral over $x_{1}$,

$$
\int_{\bar{x}_{1}=0}^{\bar{x}_{1}=1} \bar{u}_{\bar{x}_{1}} d \bar{x}_{1}=\int_{P\left(l_{0} \cap \bar{x}_{1} \text {-axis }\right)}^{P\left(l_{1} \cap \bar{x}_{1} \text {-axis }\right)}\left[u_{1}\left(x_{1}\right)-\tan \left(\frac{\Theta}{2}\right) x_{1}\right] d x_{1}+K_{0}+K_{1},
$$

where $P$ denotes projection to the $x_{1}$-axis, and $K_{0}$ as well as $K_{1}$ denotes the signed areas to the left or right of the desired region, forming the difference.

It is important to note the following for $j=1,2$ :

(i) $P\left(l_{j} \cap \bar{x}_{1}\right.$-axis $)$ is in the $x_{1}$-domain of $u_{1}$ by (4.4),

$$
\begin{aligned}
& \left|P\left(l_{0} \cap \bar{x}_{1 \text {-axis }}\right)\right| \leq 1 \cdot \cos \left(\frac{\Theta}{2}\right)<1, \\
& \mid P\left(l_{1} \cap \bar{x}_{1} \text {-axis }\right) \mid \leq(1+1) \cdot \cos \left(\frac{\Theta}{2}\right)<2 ;
\end{aligned}
$$

(ii) $P\left(l_{j} \cap \gamma\right)$ is also in the $x_{1}$-domain of $u_{1}$ as $\gamma$ is a graph over $B_{2}$,

$$
\left|P\left(l_{j} \cap \gamma\right)\right| \leq 2 ;
$$

(iii) the region $K_{j}$ is bounded by the line $l_{j}$, the vertical line $x_{1}=P\left(l_{j} \cap \bar{x}_{1^{-}}\right.$ axis), and the curve $\gamma$, also each region $K_{j}$ is on one side of the $\bar{x}_{1}$-axis.

Thus from (i)

$$
\left|\int_{P\left(l_{0} \cap \bar{x}_{1} \text {-axis }\right)}^{P\left(l_{1} \cap \bar{x}_{1} \text {-axis }\right)}\left[u_{1}\left(x_{1}\right)-\tan \left(\frac{\Theta}{2}\right) x_{1}\right] d x_{1}\right| \leq \underset{B_{2}}{\operatorname{osc}} u+C(2)
$$


and from (ii) (iii)

$$
\left|K_{j}\right| \leq\left|\int_{P\left(l_{j} \cap \bar{x}_{1} \text {-axis }\right)}^{P\left[l_{j} \cap \gamma\right]}\left[u_{1}\left(x_{1}\right)-\tan (\Theta / 2) x_{1}\right] d x_{1}\right| \leq \underset{B_{2}}{\operatorname{osc} u+C(2) .}
$$

It follows that

$$
|D u(0)| \leq C(2) \max _{\bar{B}_{1}(\overline{0})}|\bar{u}-\bar{u}(\overline{0})| \leq C(2)\left(\underset{B_{2}}{\operatorname{osc} u+1}\right) .
$$

We complete the proof of Theorem 1.2. For $\Theta \geq \pi / 4$, the bound (4.1) gives

$$
|D u(0)| \leq \underset{B_{1}}{\operatorname{osc}} u+\frac{1}{2} \leq C(2)\left[\underset{B_{2}}{\operatorname{osc}} u+1\right] .
$$

For $\Theta \leq \pi / 4$, if $\operatorname{osc}_{B_{2}} u \leq 1 /(2 \sin \Theta)$, then Proposition 4.1 gives

$$
|D u(0)| \leq C(2)\left[\begin{array}{c}
\operatorname{osc} u+1 \\
B_{2}
\end{array}\right]
$$

Otherwise, $\operatorname{osc}_{B_{2}} u>1 /(2 \sin \Theta)$, and from (4.1)

$$
|D u(0)| \leq \underset{B_{1}}{\operatorname{osc}} u+\underset{B_{2}}{\operatorname{osc}} u \leq C(2)\left[\underset{B_{2}}{\operatorname{osc}} u+1\right] .
$$

Applying this estimate on $B_{2}(x)$ for any $x \in B_{1}(0)$, we arrive at the conclusion of Theorem 1.2.

\section{REFERENCES}

[BDM] Bombieri, Enrico, De Giorgi, Ennio and Miranda, Mario, Una maggiorazione a priori relativa alle ipersuperfici minimali non parametriche. Arch. Rational Mech. Anal. 32 (1969), 255-267.

[G] Gregori, Giovanni, Compactness and gradient bounds for solutions of the mean curvature system in two independent variables. J. Geom. Anal. 4 (1994), no. 3, 327-360.

[HL] Harvey, Reese and Lawson, H. Blaine. Jr., Calibrated geometry. Acta Math. 148 (1982), 47-157.

[H1] Heinz, Erhard, On certain nonlinear elliptic differential equations and univalent mappings. J. Analyse Math. 5 (1956/1957) 197-272.

[H2] Heinz, Erhard, On elliptic Monge-Ampère equations and Weyl's embedding problem. J. Analyse Math. 7 (1959) 1-52.

[W] Wang, Mu-Tao, Interior gradient bounds for solutions to the minimal surface system. Amer. J. Math. 126 (2004), no. 4, 921-934.

[WY1] Warren, Micah and Yuan, Yu, A Liouville type theorem for special Lagrangian Equations with constraints. preprint.

[WY2] Warren, Micah and Yuan, Yu, Hessian estimates for the sigma-2 equation in dimension three. preprint.

[WY3] Warren, Micah and Yuan, Yu, Hessian estimates for three dimensional special Lagrangian equations with large phase. preprint.

[Y1] Yuan, Yu, A Bernstein problem for special Lagrangian equations. Invent. Math. 150 (2002), 117-125.

[Y2] Yuan, Yu, Global solutions to special Lagrangian equations. Proc. Amer. Math. Soc. 134 (2006), no. 5, 1355-1358. 
Department of Mathematics, Box 354350, University of Washington, SeatTLE, WA 98195, USA

E-mail address: mwarren@math.washington.edu, yuan@math.washington.edu 